\title{
Malaria among patients and aid workers consulting a primary healthcare centre in Leogane, Haiti, November 2010 to February 2011 - a prospective observational study
}

A Neuberger (a_neuberger@rambam.health.gov.il) ${ }^{1,2}, 0$ Zaulan' ${ }^{1}$, S Tenenboim ${ }^{1,3}$, S Vernet ${ }^{1}$, R Pex $^{1}, K_{\text {Held }}^{1,4}$, M Urman $^{1,5}$, K Garpenfeldt ${ }^{1}$, E Schwartz ${ }^{1,3}$

1. Israaid Primary Health Clinic, Leogane, Haiti

2. Unit of Infectious Diseases, Rambam Medical Center, Haifa, Israel

3. The Center for Geographic Medicine and Tropical Diseases, Sheba Medical Center, Tel Hashomer \& Sackler Faculty of Medicine,Tel Aviv University, Israel

4. Emergency Department, Markham Stouffville Hospital, Markham, Ontario, Canada

5. Department of Obstetrics and Gynecology, Shaare Zedek Medical Center, Hebrew University Medical School, Jerusalem, Israel

Neuberger A, Zaolan O, Tenenboim S, Vernet S, Pex R, Held K, Urman M, Garpenfeldt K, Schwartz E. Malaria among patients and aid workers consulting a primary healthcare centre in Leogane, Haiti, November 2010 to February 2011 - a prospective observational study. Euro Surveill. 2011;16(13):pii=19829. Available online: http://www.eurosurveillance.org/ViewArticle.aspx?Articleld $=19829$

Plasmodium falciparum malaria is endemic in Haiti, but epidemiological data are scarce. A total of 61 cases of malaria were diagnosed between November 2010 and February 2011 among 130 Haitian patients with undifferentiated fever. Three additional cases were diagnosed in expatriates not taking the recommended chemoprophylaxis. No cases were diagnosed among aid workers using chemoprophylaxis. In conclusion, malaria is a significant health problem in Leogane, Haiti. Aid workers and visitors should use chemoprophylaxis according to existing guidelines.

\section{Introduction}

Plasmodium falciparum malaria is endemic in Haiti [1-5]. Epidemiological data from Haiti are scarce, but before 2010 the prevalence of malaria in most areas of Haiti was estimated to be low [2-5]. The effects of the 2010 earthquake and the severe flooding that followed the 2010 hurricane on the incidence of malaria are unknown. We report the incidence of malaria among febrile patients in two primary-care clinics in the West (Ouest) Province of Haiti. In addition we report all cases of malaria in expatriate aid workers seen in our clinic.

\section{Methods}

The study was conducted in two newly established primary healthcare clinics in the West Province of Haiti. The main clinic is situated in the town of Leogane, 30 $\mathrm{km}$ west of Port-au-Prince. Leogane has an estimated population of 200,000 . The other clinic is situated in Magandou, a rural village in the same region. Since November 2010 the Leogane clinic has been operating daily, and the Magandou clinic is open once a week. Both clinics are staffed by nurses and doctors from Haiti, Israel, and Canada. Medical services are provided free of charge. All cases of undifferentiated fever were tested for malaria. Diagnoses of malaria were reached with the help of a rapid diagnostic test for detection of histidine-rich protein II (Paracheck Rapid Test, Orchid Biomedical Systems). The tests were performed in both clinics by the same experienced doctors using the same diagnostic kits. The clinical and epidemiological features of all cases of malaria were collected prospectively.

\section{Results}

Over a period of 14 weeks, between November 2010 and February 2011, a total of 61 cases of falciparum malaria were diagnosed among Haitian patients in the Leogane clinic. This period roughly correlates with the peak malaria transmission season in Haiti [3]. These 61 cases accounted for $46.9 \%$ of the 130 patients with undifferentiated fever, and $1.9 \%$ of all 3,166 patient visits. The average age of the patients with malaria was 22.5 years (range 3 to 67 years) with 25 of 61 cases occurring in patients younger than 16 years. Thirtytwo cases occurred in females. All malaria cases were acquired in Leogane, as none of the patients had travelled outside the Leogane area during the three weeks preceding the onset of symptoms.

All patients with malaria reported a febrile disease; although upon presentation only 43 of 61 had a fever higher than $37.5^{\circ} \mathrm{C}$. Two patients had severe malaria and were transferred to a referral hospital. Nearly all patients (60 of 63) were treated with chloroquine. Three patients were treated with artemether/lumefantrine; two because of difficulty in accurately dividing the chloroquine pills for young children, and one because of an allergic reaction to chloroquine. 
No cases of malaria were found among a total of 258 patients examined in the village of Magandou. Eleven of these patients had presented with an undifferentiated fever.

Three expatriates diagnosed with malaria were aid workers living in Leogane. None of the three were using anti-malaria chemoprophylaxis. Since the total number of aid workers residing in the area of Leogane is unknown, the risk of acquiring malaria in this population can not be calculated. In our organisation two out of the ten aid workers who stayed in Haiti for a total of 57 person-weeks and did not use chemoprophylaxis contracted malaria. No cases of malaria occurred in 52 additional aid workers who stayed in Haiti for a total of 346 person-weeks and used chemoprophylaxis with chloroquine.

\section{Discussion}

Studies before the earthquake reported a low risk of acquiring malaria in most areas of the country [2-5]; data from Leogane itself were not available. According to our data, collected after the earthquake and hurricane of 2010, the incidence of malaria among patients with undifferentiated fever in Leogane, Haiti was around $47 \%$. Although the sensitivity of the Paracheck Rapid Test has been reported to be sub-optimal [6], its specificity is very high. Therefore we think that the number of malaria cases has not been overestimated. A recent report from a post-earthquake national surveillance system indicated that suspected malaria and fever of unknown cause accounted for $10.3 \%$ and $10 \%$, respectively, of total visits to 51 pre-specified clinics [1]. Although laboratory diagnoses of malaria were not performed, these results seem to indicate that the incidence of malaria in certain parts of post-earthquake Haiti may be appreciable.

In a study published in 1995 only $4 \%$ of peripheral blood smears taken from febrile patients in several different provinces of Haiti were positive for $P$. falciparum [4]. It is not known whether the incidence of malaria among febrile patients was underreported in the past, or whether the natural disasters that recently affected the country have caused an increase in malaria incidence. It is also unclear whether the incidence of malaria in other areas of the country is similar to the one in Leogane. Leogane is situated near the epicenter of the 2010 earthquake. Approximately $80 \%$ of Leogane was destroyed, and tens of thousands of its inhabitants were made homeless. Since Anopheles albimanus, the mosquito vector of malaria in Haiti, usually bites outdoors, people living in temporary shelters are probably at an increased risk of contracting malaria in postearthquake Haiti. In addition hurricane Tomas caused severe floods in Leogane in November 2010, and may therefore have expanded the breeding sites for the vector.

In contrast, no cases were found in Magandou, located in the hilly areas $25 \mathrm{~km}$ south-west of Leogane. The elevation of Magandou (941 meters above sea level) does not fully explain this finding. The reasons for such a significant regional variation in the incidence of malaria within a relatively small area are unclear. Possible explanations include a more mountainous terrain, and less damage caused by both the 2010 earthquake and hurricane Tomas.

Not surprisingly cases of malaria also occurred among aid workers residing in the Leogane area. Cases of malaria among emergency responders after the 2010 earthquake were reported in other areas in Haiti, too, but since data regarding incidence are unavailable, a comparison of the risk of infection in different areas is impossible [7]. It is important to note that no cases of malaria were detected among aid workers receiving chloroquine chemoprophylaxis. Apparently, the risk of acquiring malaria in expatriates using chemoprophylaxis is appropriately low.

In conclusion, malaria is a significant health problem in Leogane, Haiti. It is unknown whether this holds true for other areas of Haiti. It is also unclear whether the high malaria incidence among febrile patients was underreported in the past, or whether it is related to the deteriorated infrastructure of the area following the earthquake and the hurricane that occurred in 2010. Aid workers and visitors should use chemoprophylaxis according to existing guidelines. We have not detected any cases of chloroquine chemoprophylaxis failure, thus supporting the current malaria prevention guidelines [8]. Further entomologic surveys and vector control efforts are warranted if malaria incidence is to be reduced in Leogane, Haiti.

\section{References}

1. Centers for Disease Control and Prevention (CDC). Launching a National Surveillance System After an Earthquake -.- Haiti, 2010. MMWR Morb Mortal Wkly Rep. 2010;59(30):933-8.

2. World Health Organization Information Resource Centre, Communicable Diseases. International Travel and Health. Country List: vaccination requirements and malaria situation. Geneva: WHO; 2005. p. 166. Available from: http://whqlibdoc. who.int/publications/2005/9241580364_country_list.pdf

3. Eisele TP, Keating J, Bennett A, Londono B, Johnson D, Lafontant C, et al. Prevalence of Plasmodium falciparum infection in rainy season, Artibonite Valley, Haiti, 2006. Emerg Infect Dis. 2007;13(10):1494-6.

4. Kachur SP, Nicolas E, Jean-François V, Benitez A, Bloland PB, Saint Jean $Y$, et al. Prevalence of malaria parasitemia and accuracy of microscopic diagnosis in Haiti, October 1995. Rev Panam Salud Publica. 1998;3(1):35-9.

5. Raccurt C. Malaria in Haiti today. Sante. 2004;14(4):201-4. [French].

6. Centers for Disease Control and Prevention (CDC). Rapid diagnostic tests for malaria ---Haiti, 2010. MMWR Morb Mortal Wkly Rep. 2010;59(42):1372-3.

7. Centers for Disease Control and Prevention (CDC). Malaria acquired in Haiti - 2010. MMWR Morb Mortal Wkly Rep. 2010;59(8):217-9.

8. Tan KR, Mali S, Arguin PM. Malaria Risk Information and Prophylaxis, by Country. In: CDC Health Information for International Travel. Atlanta: US Department of Health and Human Services, Public Health Service; 2007.. Atlanta: US Department of Health and Human Services, Public Health Service, 2007. Available from: http://wwwnc.cdc.gov/travel/ yellowbook/2010/chapter-2/malaria-risk-information-andprophylaxis/Haiti.aspx 